\title{
TRADICIÓN DISCURSIVA Y AUTORIDAD FILOLÓGICA EN LA PRIMERA PARTE DE LOS COMENTARIOS REALES DE LOS INCAS
}

\author{
DISCURSIVE TRADITION AND PHILOLOGICAL \\ AUTHORITY IN THE FIRST PART OF THE \\ REAL INCA COMMENTS
}

Percy Gerardo Prado Salazar ${ }^{1}$

\begin{abstract}
RESUMEN
En este artículo se revisará algunas de las tradiciones del discurso identificadas en el texto de la primera parte de los Comentarios Reales. Se repasará la huella de la retórica de la época, específicamente en cuanto al género histórico de los comentarios y la relación notarial. Tras la revisión de ambos aspectos se los vinculará con la construcción de autoridad filológica del Inca Garcilaso de la Vega y con la importancia, en el fondo de todo esto, de la palabra como medio para sostener la visión reconciliadora y armonizadora planteada por el cronista mestizo.
\end{abstract}

Palabras clave: Comentarios Reales, Inca Garcilaso, discurso, filología, historia.

\section{ABSTRACT}

This article will review some of the discourse traditions identified in the text of the first part of the Royal Commentaries. The rhetoric of the period will be reviewed, specifically regarding the historical gender of the comments and the notarial relationship. After reviewing both aspects, they will be linked with the construction of philological authority of the Inca Garcilaso de la Vega and with the importance, at the heart of all this, of the word as a means to sustain the reconciling and harmonizing vision posed by the mestizo chronicler.

Keywords: Real Comments, Inca Garcilaso, speech, philology, history.

\section{INTRODUCCIÓN}

Los estudiosos de la obra del Inca Garcilaso de la Vega coinciden en que elaboró sus Comentarios Reales de manera minuciosa. Solo en la escritura de la primera parte (es decir, dejando a un lado la Historia general del Perú) invirtió, por lo menos, nueve años. Según José Durand, «hacia 1604 [los Comentarios reales] estaban ya casi al fin» $(1976$, p. 151) y habría que retroceder hasta 1596 para marcar el inicio de su redacción, como lo confiesa en el manuscrito de la Relación de la descendencia de Garci Pérez de Vargas cuando revela su

'Pontifícia Universidad Católica del Perú. Lima-Perú. E-mail: percygerardops@gmail.com

Presentado: 27/03/18 Aprobado: 25/06/18 
intención de tratar sobre «la historia del origen y descendencia de aquellos Reyes Incas» a cuya escritura pasará «luego que quitemos la mano de esta historia» (2015 [1596], p. 372).

Garcilaso proyectó escribir los Comentarios desde 1586, año en que firma la dedicatoria de su traducción de Diálogos de amor de León Hebreo, donde confiesa que ya los tenía bosquejados; en tanto que en 1605 «Damiam Daguiar y Costa [conceden la Licencia del Paso] a los "Comentarios Reales de los Incas", pero el Inca Garcilaso solo editará ese año [ $L a$ Florida]» (González-Vigil, 2007: LXVII, comillas del autor). En buena cuenta son cerca de dos décadas de trabajo que demuestran el nivel de cuidado en la elaboración discursiva de la primera parte de los Comentarios Reales. La escritura y el estudio diligentes terminaron por hacer de la obra del Inca Garcilaso de la Vega un texto de gran interés no solo por la elegancia de su prosa y su arreglo formal, sino también por sus novedades textuales como la construcción de su autoridad discursiva, las resonancias andinas quechuas $\mathrm{y}$, sobre todo, debido a su apuesta por la reconciliación de dos culturas enfrentadas, conflicto que el propio autor vivió en carne propia.

La vida del cronista cusqueño tiene también para los críticos mucha importancia. Su condición de mestizo en un entramado social marcado por el choque de dos culturas ha llevado a muchos a proyectar en su figura y en la de su obra, la idea de una nación reconciliada con sus "dos mitades"; es decir, la idea utópica de la patria mestiza armónica.

La vida y obra del Inca parecen constituir un conjunto de estímulos sobre los cuales se puede imponer los significados que les atribuye el observador. Como en un test de Rorschach, los datos están ahí presentes. Se puede responder a ellos como si se tratase de una prueba proyectiva [...]. El Inca se transforma según los ámbitos ideológicos por los que circula y de acuerdo a las perspectivas personales de sus lectores. Un solo personaje verdadero en tres versiones distintas (Hernández, 1991, pp. 170-171).

Como lo señala Max Hernández, en muchas ocasiones, las lecturas del Inca Garcilaso de la
Vega han resultado más bien proyecciones de los deseos del investigador. Para decirlo parafraseando una idea neoplatónica, a las que tan cercano era el Inca, el objeto conocido fue apreciado en función (y fruición) del sujeto cognoscente (v. Boecio, La consolación de la filosofia, libro V, prosa 4). Las «tres versiones distintas» de las que se habla son lo español, lo indio y lo mestizo que encarna Garcilaso. Así, en la trayectoria crítica de su obra ha habido quienes idealizaron la prosapia hispana, como José de la Riva-Agüero; la condición india, como Luis E. Valcárcel; y lo mestizo como Luis Alberto Sánchez, quien lo llama «el primer cholo significativo del Perú y de América» (1979, p. 10). Las idealizaciones a partir de la obra del Inca mezcladas con reivindicaciones de clases son recurrentes entre los garcilasistas de las primeras décadas del siglo pasado. Hacia mediados del siglo, tras los aportes de Aurelio Miro Quesada y José Durand, se va a imponer la figura del Garcilaso trágico y mestizo, lo que - según César Delgado Díaz del Olmo- se «corresponde [con el] angustioso despertar de la conciencia del mestizo de los grandes sectores populares emigrantes que comienza[n] a interrogarse sobre su identidad» $(2009$, p. 78$)$. El estudio de la recepción crítica de la obra garcilasiana es un campo interesante sobre el cual debería haber mayores trabajos ${ }^{2}$. En épocas distintas, la vida y la obra del Inca han presentado distintas y nuevas interpretaciones $e$ interpelaciones; son una fuente de estudio que no solo sirve para acercarse a su tiempo, sino también para echar luces sobre los contextos en los que fue leído.

En este trabajo se ofrecerá un acercamiento crítico y objetivo en la medida de lo posible a las tradiciones discursivas occidentales que concurren en el texto de la primera parte de los Comentarios Reales publicada en Lisboa en 1609. La atención recaerá sobre el género histórico denominado comentarios, al cual pertenece la obra mayor del Inca Garcilaso y sobre algunas características que comparte con la relación notarial. El objetivo es, tras el repaso de las fundamentaciones críticas que sostienen

\footnotetext{
${ }^{2}$ Un artículo que trata el tema es «Lectura y difusión de la obra del Inca Garcilaso en el virreinato peruano (siglos XVII-XVIII): El caso de los Comentarios Reales» de Pedro Guibovich, publicado en Revista histórica, tomo 37 (19901992), pp. 103-120.
} 
dicha peculiaridad textual, describir el modo como el cronista valida su autoridad histórica en base a razones filológicas y relacionarlo con la confianza del Inca en la potencia de la lengua y la palabra para sostener su propuesta armonizadora de cuño neoplatónico.

\section{Los comentarios como género histórico} Desde el título, los Comentarios Reales ${ }^{3}$ hacen referencia a un género histórico tradicional. Al respecto, señala Raúl Porras Barrenechea:

Entre las diversas formas historiográficas adoptadas por la historia clásica -historias, anales, memorias, comentarios- la elegida por el Inca es la de menor categoría. "Comentarios, dice Cicerón, son simples notas conmemorativas" [...]. Los comentarios son breves notas o glosas a noticias ajenas que no requieren gran ingenio ni preparación. En ellos se limitará a glosar a los historiadores españoles que han escrito sobre su patria sirviéndoles únicamente de "comento y glosa", corrigiendo o ampliando lo que ellos dijeron, aclarando lo que no supieron $o$ no pudieron saber por su desconocimiento de la lengua y añadiendo, donde hubiere falta. (1946, pp. 10-11)

Para el historiador peruano, la obra de Garcilaso se adscribe en el género menor de los comentarios. Del mismo modo, aunque incidiendo en su parecido con el título y el número de capítulos del libro de Julio César, Comentarios de la guerra de Las Galias, Aurelio Miro Quesada y Carlos Daniel Valcárcel señalan la pertenencia de los Comentarios Reales al mismo tipo de discurso histórico (Fernández, 2004,pp. 26-27).

Con respecto a la huella de Julio César en el Inca, Margarita Zamora rebate dicha posibilidad en su trabajo Language, Authority, and Indigenous History in the Comentarios

\footnotetext{
${ }^{3}$ El título completo es Primera parte de los commentarios reales, que tratan del origen de los Yncas, reyes quefveron del Perv, de sv idolatría, leyes y gouierno en paz y en guerra: de sus vidas y conquistas, de todo lo que fue aquel Imperio y su República, antes que los Españoles passaran a él.
}

Reales de los Incas de 1988. Estrella Guerra, quien en su tesis toma algunas de las premisas de Zamora, refuta esta idea al demostrar la relación textual de imitación entre ambas obras (1996, pp. 129-159). Por su parte, Christian Fernández señala la inexactitud de Zamora al asumir «que los Comentarios de Julio César pertenecen al género de comentario histórico que ella describe» (2004, p. 32).

El Inca Garcilaso no fue inmune a la influencia de la retórica de su tiempo; todo lo contrario, alcanzó un manejo magistral en el arte de la escritura histórica. Miguel Lasso de la Vega, Marqués del Saltillo, publicó en 1929 la Relación de la descendencia del famoso Garcí Pérez de Vargas en la Revista de Historia y de Genealogía Española número 16 y en el mismo lugar reveló la dedicatoria al Inca Garcilaso hecha por Francisco de Castro en su libro De Arte Rhetorica, «en un acto de reconocimiento a su calidad como retórico y como historiadon» (Guerra, 1996, p. 60). La contundencia de este hecho sirve para mostrar el respeto y admiración que había logrado el Inca en el círculo intelectual de Montilla y Córdoba que frecuentaba.

Estrella Guerra, en el capítulo II de su investigación ya citada, constata la relación de aplicación entre los Comentarios Reales y el libro de instrucción retórica $D e$ ratione dicendi (1532) de Juan Vives (1492-1540 $)$. En este tipo de relación se «pone en práctica lo que ahí [en el libro de Vives] se propone como el óptimo discurso historiográfico» (Guerra, 1996, p. 162). Ambos hechos, la dedicatoria en un libro de retórica y la aplicación de un modelo de discurso histórico, demuestran no solo la influencia de la retórica de su época, sino también prueban el domino alcanzado por el Inca en cuanto al manejo discursivo. Entonces, no es un dato falto de importancia el que Garcilaso haya elegido para su obra el título de Comentarios reales, este tipo de texto histórico le permitía cumplir a cabalidad con su objetivo de comentar, ampliar y corregir a los cronistas

\footnotetext{
"En la lista de títulos de la biblioteca del Inca Garcilaso de la Vega no aparece este libro de Juan Vives, pero sí se encuentra otro que titula Introductión a la sabiduría, editada en Amberes por Johann Steelsius en 1551. Véase el apéndice del artículo de Teodoro Hampe Martínez, José Durand, bibliófilo (su colección de libros y papeles en la universidad de Notre Dame) en Revista de Indias, 1997, vol. LVII, núm. 210 , pp. 556-562.
} 
españoles y, de ese modo, reescribir la historia, como se verá más adelante.

\section{La retórica notarial}

Roberto González Echevarría, en su libro Mitoy archivo. Una teoria de la narrativa latinoamericana (2000) sostiene que «[1]a mayoría de los lectores de los Comentarios Reales de los Incas tendrían dificultad para parangonar el libro de Garcilaso con la retórica notarial o picaresca, pero esto se debe a que generalmente solo se lee la primera parte de la obra» (p. 112).

Aunque González Echevarría incide más en la segunda parte de los Comentarios Reales, es decir, en la Historia general del Perí, extiende sus afirmaciones al conjunto de los dos libros, pues «en cierto sentido - alega-, podría decirse que la primera parte cabe dentro del diseño de la segunda y depende de ella, y no al contrario» (p. 113). Los rasgos notariales de la obra del Inca se deberían a que el motivo de su escritura fue, según precisa González Echevarría, el fallo del Consejo de Indias en 1563 en contra de las mercedes que pretendía Gómez Suárez de Figueroa ${ }^{5}$ en pago por los servicios prestados por su padre a la corona y por ser él un descendiente de sangre real inca. Fue Lope García de Castro, integrante del Consejo, quien derrumbó todas las esperanzas del joven mestizo al acusar al capitán Garcilaso de la Vega, su padre, de traicionar al rey durante la batalla de Huarina, pues cedió su caballo al rebelde Gonzalo Pizarro para que este escapara. García de Castro conoció este hecho por las crónicas del Paletino y de Gómara, a quienes el Inca citará, comentará, corregirá y desautorizará a lo largo de su obra.

González Echevarría, además de ver a los Comentarios como un texto de alegato en el que un relator somete a prueba la validez de sus argumentos, señala su analogía con la literatura picaresca en cuanto que ambos tipos discursivos serían una relación. Cabe recordar en este punto los tipos discursivos básicos presentes en los Comentarios Reales:

\footnotetext{
${ }^{5}$ «A lo largo de sus sesenta y siete años de existencia el Inca Garcilaso usó hasta cuatro nombres diferentes: Gómez Suárez de Figueroa hasta los 25 años; Gómez Suárez de la Vega durante unos meses de 1563 y Garcilaso de la Vega desde esa fecha hasta su muerte ocurrida medio siglo más tarde» (De Solano, 1991, p. 121).
}

narración, descripción y argumentación ( $\mathrm{v}$. Guerra, 1996, pp. 74-128). La narración y descripción resaltan a lo largo de la primera parte, que es la que analiza Estrella Guerra y es a la que nos referimos; en tanto que el discurso argumentativo solo es predominante en el Proemio, en las Advertencias acerca de la lengua general de los indios del Perú y en el capítulo XIX del libro I, Protestación del autor sobre la historia (Ibíd.).

[Rolena] Adorno, al estudiar 1a propuesta de González Echevarría, ha notado que, si bien los discursos narrativos de las crónicas y las relaciones comparten algunos recursos retóricos y estilísticos, hay instancias que pertenecen solo a la relación como discurso legal oficial y que no son compartidas por otros géneros discursivos. (Fernández, 2004,p. 34)

\section{La tradición filológica}

Para Margarita Zamora, los críticos que sostienen que los Comentarios Reales son el punto de partida de la literatura hispanoamericana — González Echevarría uno de ellos- tienen razón. Esta es una afirmación que merece una restricción, no se refiere Zamora al hecho _como quieren ver algunos - de que los Comentarios constituyan un discurso novelesco o imaginativo, sino que se trata de una obra cuya originalidad discursiva recae sobre su condición de metatexto, es decir, se trata de un comentario filológico sobre un texto anterior (Zamora, 1987, p. 557). ¿Cuál o cuáles son estos textos? Primero, hay que decir que los Comentarios son «a la vez exégesis correctiva y una rigurosa hermenéutica» (Id.: 555), por un lado, se trata de corregir las malas interpretaciones de los españoles sobre la cultura incaica, dado su desconocimiento de la lengua quechua y, por otro, se intenta restaurar el texto original de la historia de los Incas, gracias al dominio de la lengua quechua que posee Garcilaso, de allí que -como lo confiesa- su intención sea la de servir de «comento y glosa» de los historiadores españoles.

Siguiendo a Zamora, se debe destacar que, frente a los cronistas hispanos, el Inca construye una original postura de autoridad que revela la influencia de la filología de su tiempo. La 
estrategia de Garcilaso es la de un intérprete en busca de la traducción precisa basada en su dominio idiomático y cultural, lo que constituye la línea básica de toda estrategia filológica ( $v$. Guerra, 1996; Zamora, 1987). La obra garcilasiana está vinculada con la forma del comentario filológico bíblico, común en su tiempo y tan usado entre el círculo de jesuitas cordobeses que frecuentaba el autor mestizo (v. De Mora, 2010; Durand, 1963; Guerra, 1996; Zamora, 1987). Christian Fernández, en el primer capítulo de su libro Inca Garcilaso: Imaginación, memoria e identidad (2004), rebate la idea de que los Comentarios Reales pertenezcan al género filológico.

$[\mathrm{N}]$ o se puede negar que Garcilaso use el recurso filológico en algunos pasajes de su obra, pero afirmar que toda la obra del Inca está concebida siguiendo las características de este género es mucho decir. Además, no hay que olvidar que, a pesar de que los historiadores actuales nieguen la veracidad de algunos de los hechos tratados por Garcilaso en su obra, dentro de la concepción de la época, $l o$ que Garcilaso escribe es historia, es decir, narración de hechos de manera verosímil y no una explicación de terminología. (Fernández, 2004, p. 32, cursivas nuestras)

A Garcilaso le preocupa dar «relación entera» sobre el tiempo de los incas y de la conquista. En esta intención, incide mucho en la correcta interpretación por parte de los españoles de los «vocablos indios, que, como extranjeros en aquella lengua, interpretaron fuera de la propiedad de ella, según que largamente se verá en el discurso de la historia) (Comentarios Reales, Proemio). Garcilaso siempre hace mención a que su libro es historia y que, dentro de ella, parte fundamental es el «comento y glosa»; sobre todo, de los términos lingüísticos y fenómenos culturales quechuas (religión, gobierno, etc.) mal entendidos por los «españoles curiosos» (o estudiosos, según el significado más usado en aquel tiempo, como lo explica Sebastián de Covarrubias en 1611). No solo glosará de esto último, también lo hará sobre los hechos acaecidos, aunque parece ser consciente de que, frente al lector de su época, su autoridad como historiador puede quedar en entredicho por su misma condición de indio:
... y no escribiré novedades que no se hayan oído, sino las mismas cosas que los historiadores españoles han escrito de aquella tierra y de los Reyes de ella y alegaré las mismas palabras de ellos donde conviniere, para que se vea que no finjo ficciones en favor de mis parientes, sino que digo lo mismo que los españoles dijeron. (CR, I, 19)

\section{La autoridad del Inca}

Con respecto de la autoridad discursiva del Inca, Max Hernández mantiene que

Cuando Garcilaso se preparaba a objetivar y fijar discursivamente su realidad cultural originaria pudo compararse, ventajosamente, con quienes desde Europa; y sin poseer ni la sensibilidad ni los instrumentos cognoscitivos presentes en la tradición andina, intentaban comprender la rea 1 idad descubierta. La heterogeneidad de los dos espacios geográficos e históricos y de los modos autóctonos que cada tradición había desarrollado para la apropiación cultural de los mismos, permitía al Inca Garcilaso ostentar, en medio de su modestia mestiza de indio antártico, los emblemas de un sentimiento de superioridad. (1991, p. 169)

Efectivamente, esta superioridad del Inca le permite validarse como una voz autorizada para corregir y ampliar lo dicho por los españoles.

Sólo serviré de comento para declarar y ampliar muchas cosas que ellos asomaron a decir y las dejaron imperfectas por haberles faltado relación entera. Otras muchas se añadirán que faltan de sus historias y pasaron en hecho de verdad, y algunas se quitarán que sobran, por falsa relación que tuvieron, por no saberla pedir el español con distinción de tiempos y edades y división de provincias y naciones, o por no entender al indio que se la daba o por no entenderse el uno al otro, por la dificultad del lenguaje. Que el español que piensa que sabe más de él, ignora de diez partes las nueve por las muchas cosas que un mismo vocablo significa y por las diferentes pronunciaciones 
que una misma dicción tiene para muy diferentes significaciones, como se verá adelante en algunos vocablos, que será forzoso traerlos a cuenta. (CR, I, 19 , cursivas nuestras)

En el Tesoro de la lengua castellana o española de Sebastián de Covarrubias se puede leer el único significado que "declarar» tenía entonces: «manifestar lo q de suyo estava oculto obscuro, y no entendido» $(1611$, p. 599, 1). En ese sentido, Garcilaso fundamenta su autoridad como comentarista de los «españoles curiosos» en conocer la lengua quechua por ser indio Inca que habla en español, este es el sentimiento de superioridad del que habla el psicoanalista Max Hernández: la validación textual como historiador relacionada también con lo visto y lo vivido. Pero los Comentarios Reales no son una simple fantasía psicoanalítica compensatoria, su obra está fuertemente determinada por las estructuras retóricas de la época, si de algún modo iba a aflorar lo andino debía de ser en momentos de crisis, en los lugares donde el discurso occidental se fracture. Los Comentarios Reales se evidencian más bien como el más alto ejemplo de cómo un hombre nacido en tierras antárticas, un mestizo a boca llena, con la sola fuerza de su voluntad e inteligencia alcanzó y perfeccionó el manejo de las herramientas retóricas occidentales para usarlas en su favor y contra los estudiosos españoles, especialmente contra aquellos que se oponían a las leyes naturales que reconocían el derecho de los incas como verdaderos reyes del riquísimo y grande imperio llamado Perú.

\section{Conclusión: reconciliación de palabra}

En el Proemio de los Comentarios reales, el Inca anuncia su misión de servir como intérprete entre dos mundos encontrados: el andino y el español. Sobre los cronistas españoles señala que su

intención no es contradecirles, sino servirles de comento y glosa, y de intérprete en muchos vocablos indios, que como extranjeros en aquella lengua interpretaron fuera de propiedad de ella. (CR, I, 19)

Garcilaso emprende el desarrollo de su obra en aras de una auténtica interpretación de la lengua quechua, cuestión que ve como necesaria para entender la verdad histórica ante la presentación distorsionada, errada que ofrecen los demás cronistas. Su objetivo sobrepasa el deseo de solo comentar y corregir lo dicho en otras obras históricas. A diferencia de los otros autores, Garcilaso presenta la Conquista como un hecho sin carga traumática y sitúa la historia del incario en el horizonte de la historia del occidente cristiano.

Acuérdome que un día, hablando aquel inca viejo en presencia de mi madre dando cuenta de estas cosas y de la entrada de los españoles y de cómo ganaron la tierra, le dije:

-Inca, ¿cómo sido esta tierra de suyo tan áspera y fragosa y siendo vosotros tantos $y$ tan belicosos y poderosos para ganar y conquistar tantas provincias y reinos ajenos dejasteis perder tan presto vuestro imperio y os rendisteis a tan pocos españoles?

Para responderme volvió a repetir el pronóstico acerca de los españoles -que días antes lo había contado-y dijo cómo su inca les había mandado que los obedeciesen y sirviese, porque en todo se les aventajarían. Habiendo dicho esto se volvió a mí con gran enojo de que se les hubiese motejado de cobardes y pusilánimes y respondió a mi pregunta diciendo:

-Estas palabras que nuestro inca nos dijo, que fueron las últimas que nos habló, fueron más poderosas para sujetarnos y quitar nuestro imperio que no las arma de tu padre y sus compañeros trajeron a esta tierra. $(\mathrm{CR}$, IX, 15)

En este pasaje aparece claramente el diseño providencialista del Inca. No se trata de dos mundos enfrentados, ambos forman parte de un solo mundo, la historia de los incas es parte de la historia universal y cristiana; las palabras de Huayna Cápac sujetaron más a los belicosos incas que las armas de los españoles, pues la historia estaba así diseñada por la providencia y porque, además, la palabra tiene la potencia de ofrecer una síntesis conciliadora a la que Garcilaso apelará a lo largo de su obra. Por eso es tan importante para él la correcta interpretación de la lengua quechua, el real sentido de las palabras de los incas. Nadie como él - un indio que mamó el quechua en la leche materna, un mestizo a boca llena, un traductor 
humanista y neoplatónico- podía revelar a los españoles de su tiempo el verdadero significado y la verdadera posición de los incas en la historia del mundo.

\section{Obras del Inca Garcilaso de la Vega consultadas}

Relación de la descendencia de Garci Pérez de Vargas (2015 [1596]). En Obras completas. Edición y notas de Carlos Araníbar. Lima: Biblioteca del Perú, Colección Bicentenario Ministerio de Relaciones Exteriores. Tomo I: 355-385. También en Obras completas, tomo I, Biblioteca de Autores Españoles, 1965, pp. 230 ss., Madrid: Atlas, versión digitalizada descargada el 20 de mayo de 2017 a las 07:14 horas de

http://www.eumed.net/fuaig/docs/descendenci a.pdf.

Comentarios Reales de los Incas. Lima: Fondo Cultura Económica, edición, prólogo, índice analítico y glosario de Carlos Araníbar, 2005.

\section{REFERENCIAS BIBLIOGRÁFICAS}

COVARRUBIAS, S. (1611) Tesoro de la lengua castellada o española. Consultado el 2 de julio de 2017 a las 05:37 horas en http://buscon.rae.es/ntlle/SrvltGUILogi nNtlle

DELGADO, César (2009) El inca mestizo. Reedición corregida y aumentada de Diálogo de los mundos. Ensayo sobre el Inca Garcilaso de la Vega, 1991. Arequipa: Centro de EdicionesEditorial UNSA.

DE MORA, Carmen (2010) "La amistad del Inca Garcilaso con los humanistas de Córdoba". En Renacimiento mestizo: Los 400 años de los Comentarios reales, José Antonio Mazzoti (ed.), Madrid: Iberoamericana.

DE SOLANO, Francisco (1991) "Los nombres del Inca Garcilaso: Definición e identidad". En Anuario de Estudios Americanos, vol. 48: 121-150.

DURAND, José(1963)(1976) El nombre de los Comentarios Reales. En Revista del Museo de la Nación, pp. 322-332, Lima. El Inca Garcilaso, clásico de América.
México: Editorial Septentas.

FERNÁNDEZ, Christian 2004 Inca Garcilaso: Imaginación, memoria e identidad. Lima: Fondo Editorial Universidad Nacional Mayor de San Marcos. Versión digital descargada el 20 de mayo de 2017 a las 21:11 horas de http://sisbib.unmsm.edu.pe/bibvirtual/li bros/historia/Inca\%20Garcilaso imagi nacion/contenido.htm

GUERRA, Estrella (1996) La construcción discursiva de los Comentarios Reales de los Incas en el contexto de la retórica de su tiempo. Tesis para licenciatura, Pontificia Universidad Católica del Perú.

GONZÁLEZ E., Roberto (2000) Mito y archivo. Una teoría de la narrativa latinoamericana, México: Fondo de Cultura Económica, 2011.

GONZÁLEZ Vigil, Ricardo (2007) Prólogo y cronología. En Comentarios Reales de los Incas, Inca Garcilaso de la Vega, Lima: Fondo editorial de la Universidad Inca Garcilaso de la Vega, pp. XIXLXXVIII.

HAMPE, Teodoro (1997) José Durand, bibliófilo (su colección de libros y papeles en la Universidad de Notre Dame). En Revista de Indias, vol. LVII, núm. 210,pp. 556-562.

HERNÁNDEZ, Max (1991) Memoria del bien perdido. Conflicto, identidady nostalgia en el Inca Garcilaso de la Vega. España: Ediciones Ciruela.

PORRAS B., Raúl (1946) El Inca Garcilaso de la Vega (1539-1616). Lima

SÁNCHEZ, Luis Alberto [1939] 1979 Garcilaso Inca de la Vega primer criollo. Lima: Editorial Eusonia Talleres.

ZAMORA, Margarita. (1987) "Filología humanista e historia indígena en los Comentarios Reales". En Revista Iberoamericana, vol. LIII, núm. 140, julio-septiembre. Versión digital descargada el 25 de mayo de 2017 a las 22:31 horas de https://revistaiberoamericana.pitt.edu/ois/index.php/I beroamericana/article/view/4349 